\title{
The image series forgery detection algorithm based on the camera pattern noise analysis
}

\author{
N I Evdokimova ${ }^{1}$ and V V Myasnikov ${ }^{1,2}$ \\ ${ }^{1}$ Samara National Research University, Moskovskoe Shosse 34A, Samara, Russia, 443086 \\ ${ }^{2}$ Image Processing Systems Institute of RAS - Branch of the FSRC "Crystallography and \\ Photonics" RAS, Molodogvardejskaya street 151, Samara, Russia, 443001 \\ e-mail: nadezh.evdokimova@gmail.com,vmyas@geosamara.ru
}

\begin{abstract}
In the paper, the image series forgery detection algorithm based on the analysis of camera pattern noise is proposed. Distribution characteristics of the camera pattern noise are obtained by extracting the noise component of images from the non-tampered image series. A noise residual of a forgery image is compared with the camera pattern noise. We compare various noise filtering algorithms to choose the one that achieves the best performance of the proposed method. The proposed algorithm is tested both on examples of copy-move forgeries and forgery fragments which were inserted from an image not included in the image series.
\end{abstract}

\section{Introduction}

Image time series describes a scene dynamic. Analysis of image series allows predicting an image that may be next in the image series, as well as to conclude the authenticity of the image. There are several approaches to detect forged images. These approaches can use temporal and spatial correlations [1], unique artifacts of compression, and, finally, unique artifacts left by the camera. Methods using temporal and spatial correlations are divided into two categories. The methods belonging to the first category are based on pixel analysis of images [2-5] while the methods from the second category use the object level of images [6].

In the conditions of availability of many graphic editors and ease of their use, even an ordinary user does not require specialized knowledge and skills to falsify images. Forgeries can be made to add a new object to the scene captured by the camera or to hide the existing ones. Image series forgery detection has its distinctive features as compared to images matching since each image of an image series captures a scene at different moments. Two neighboring images of an image series can be captured under different lighting, weather or seasonal conditions. This paper proposes a forgery detection algorithm that is invariant to the conditions for obtaining images of a series.

This work consists of three parts. The first part deals with the model of the camera sensor noise and presents a method for extracting pattern noise. In the second part of the work, an algorithm for image forgery detection is proposed. The third part contains an experimental result of the proposed algorithm effectiveness. Experiments are focused on copy-move detection (fragments duplicated within one image) and copy-paste detection (fragments inserted from an image not included in the image series). 


\section{Model of a camera's sensor noise}

When the camera sensor captures a uniformly lit scene, the output image will contain a certain number of pixels, slightly different in brightness from the rest. This fact is related to random noise components, such as readout noise (the magnitude of the matrix signal fluctuations relative to the average signal value) or shot noise (random fluctuations of voltages and currents relative to their average value), and a deterministic component - pattern noise. Pattern noise is present in each image, fixed by the sensor, and remains approximately the same for different images captured by the sensor.

The output image of the camera can be represented as follows [7]:

$$
y(i, j)=f_{i, j}(x(i, j)+\eta(i, j))+c(i, j)+\varepsilon(i, j)
$$

where $\eta=\eta(i, j)$ is shot noise, $\varepsilon=\varepsilon(i, j)$ is readout noise, $c=c(i, j)$ is fixed pattern noise (FPN), $x=x(i, j)$ is image of the scene in the absence of any noise and $f_{i, j}$ is a multiplicative coefficient characterizing photo-response nonuniformity (PRNU).

\subsection{Extraction of sensor pattern noise}

To reduce the contribution of the random noise $\varepsilon=\varepsilon(i, j)$ and $\eta=\eta(i, j)$ to the determined noise component, an image time series $I_{i}(n, m), i=\overline{1, L}, n=\overline{1, N}, m=\overline{1, M}$, of the same scene captured by the same camera is used.

A $F$ noise filter is used [8], [9] to extract the high-frequency component of camera noise. For each image of the sequence $I_{i}(n, m)$, it is possible to define the pattern noise matrix $W_{i}(n, m)$ as follows:

$$
W_{i}(n, m)=I_{i}(n, m)-F\left(I_{i}(n, m)\right) .
$$

Estimation of pattern noise matrices $W_{1}(n, m), W_{2}(n, m), \ldots, W_{L}(n, m)$ set can be performed using a matrix of per-element expected values and a matrix of per-element dispersion values. These matrices can be calculated using (3) and (4), respectively. Both the matrix of expected values and the matrix of dispersion values have the same dimensions and depth as the pattern noise matrix $W_{i}(n, m)$ and the original images $I_{i}(n, m)$ accordingly.

$$
\begin{gathered}
E\left\{W_{0}, W_{1}, \ldots, W_{L}\right\}=E(n, m)=\frac{1}{L} \cdot \sum_{i=0}^{L-1} W_{i}(n, m) . \\
D\left\{W_{0}, W_{1}, \ldots, W_{L}\right\}=D(n, m)=\frac{1}{L} \cdot \sum_{i=0}^{L-1}\left(W_{i}(n, m)-E(n, m)\right)^{2} .
\end{gathered}
$$

Expected values and dispersion are calculated for every pixel of every dimension.

\subsection{Selection of a noise extraction filter}

The main requirement for a noise filter is the high quality of filtering areas around the edges of objects. This requirement is imposed so that the noise matrices contain the least amount of scene traces. The median filter, the Lee filter [10], the Gauss filter, the non-local mean filter [11] and the bilateral filter were chosen in the work.

\section{Forgery detection algorithm}

After obtaining pattern noise distribution characteristics of the camera, the noise component of the suspicious image distortion is extracted. Let $I_{F}(n, m)$ be a suspicious image that captures the same scene with the same camera. The image is not included in the image series used in (3) and (4). The pattern noise matrix of the suspicious image is determined as follows:

$$
W_{F}(n, m)=I_{F}(n, m)-F\left(I_{F}(n, m)\right) .
$$

The image forgery detection algorithm can be introduced as follows:

- Obtaining pattern noise distribution characteristics of the camera using an image series;

- Obtaining the pattern noise matrix of the suspicious image. It is needed to use the same noise filter with the same parameters; 
- Evaluation of similarity between the pattern noise of the suspicious image and the pattern noise of the camera;

- Creating of binary mask and post-processing of it.

Pattern noise distribution characteristics of the camera are obtained in the way described in the previous part of this work.

\subsection{Calculation of similarity between the noise of suspicious image and camera pattern noise}

The pattern noise matrix of the suspicious image $W_{F}(n, m), n=\overline{1, N}, m=\overline{1, m}$ is calculated using the formula (2). If images of the image series $I_{i}(n, m), i=\overline{1, L}$, have three channels, then every element of the pattern noise matrix can be presented as a vector $\mathbf{w}_{i, j}=\left(w_{i j}^{R}, w_{i j}^{G}, w_{i j}^{B}\right)^{T}, i=\overline{1, N}, j=\overline{1, M}$. The similarity between the noise of the suspicious image and camera pattern noise distribution is characterized by the Mahalanobis distance. The Mahalanobis distance is calculated for every element $\mathbf{w}_{i, j}$ of pattern noise matrix and the corresponding element $\boldsymbol{\mu}_{i, j}=E(i, j)=\left(\mu_{i j}^{R}, \mu_{i j}^{G}, \mu_{i j}^{B}\right)^{T}$ of expected values matrix using (6).

$$
d_{M}\left(\mathbf{w}_{i, j}, \boldsymbol{\mu}_{i, j}\right)=\sqrt{\left(\mathbf{w}_{i, j}-\boldsymbol{\mu}_{i, j}\right)^{T} \mathbf{B}_{i j}^{-1}\left(\mathbf{w}_{i, j}-\boldsymbol{\mu}_{i, j}\right)},
$$

where $\mathbf{B}_{i j}$ is a covariance matrix.

Set of Mahalanobis distance $d_{M}\left(\mathbf{w}_{i, j}, \boldsymbol{\mu}_{i, j}\right)$ calculated for every $\mathbf{w}_{i, j}$ forms a Mahalanobis distance matrix $\mathbf{D}_{M}\left\{\mathbf{W}_{F}, \mathbf{E}\right\}$. Next, the matrix $\mathbf{D}_{M}$ is averaged in the window whose size does not exceed the size of the forged region to carry off peak values caused by random noise.

\subsection{Creating a binary mask based on the distance matrix}

The task of creating a binary mask is solved by choosing a threshold and threshold processing on the Mahalanobis distance matrix.

The threshold is selected based on the analysis of the Mahalanobis distance matrices total histogram. The cumulative histogram is created by aggregating Mahalanobis distance matrices histograms of authentic images $I_{i}(n, m), i=\overline{1, L}$.

Neyman-Pearson criterion is used to select a value of the threshold $T$. The probability of a falsepositive $p_{0}$ is fixed, and the value of the threshold $T$ is chosen to minimize the probability of a falsenegative $p_{1}$.

\subsection{Binary mask post-processing}

In the work, post-processing of a binary mask includes selecting connected regions on the mask [12] and filtering them by size. A connected area is considered forged if its square exceeds $1 / 1000$ of the original image square. A minimal convex hull is constructed around each of forged region. Then the space inside the minimal convex hulls is filled.

\section{Experiments}

The experiments were carried out on a standard PC (Intel Core i5-4460, 16 GB RAM).

Ten image time series were used as the object of experiments. Every image series includes 15 authentic images and two forged images. All images were represented in the RGB space. One forged image included a copy-move and a fragment of another image was inserted into the second image. All images had a size of $4032 \times 3024$.

Figure 1 illustrates the camera pattern noise extracted by: (a) - the median filter, (b) - the Lee filter, (c) - the Gauss filter, (d) - the non-local mean filter and (e) - the bilateral filter. The images of camera pattern noise have been converted to grayscale and transformed by linear enhancement to the range of $[0,255]$. 
a)

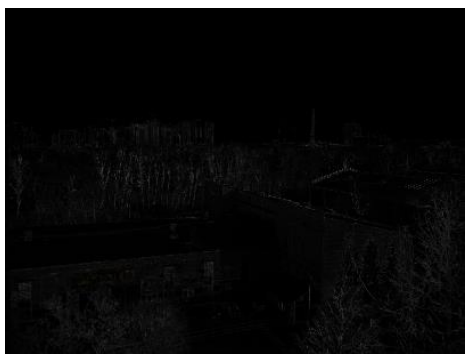

b)
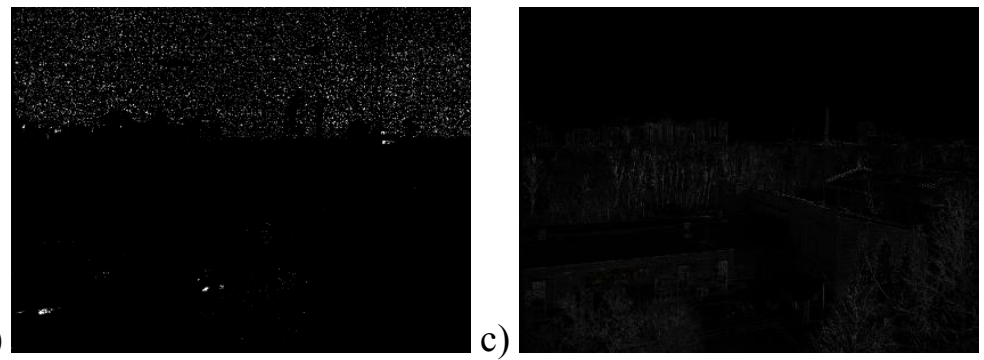

d)
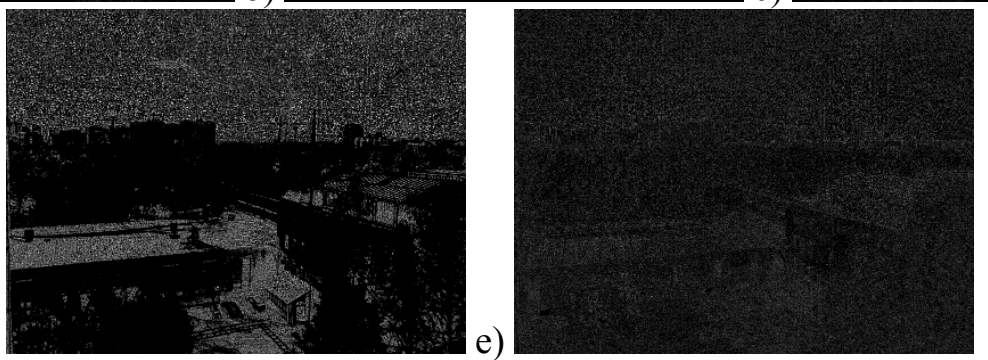

Figure 1. Camera pattern noise extracted by: $a$ - the median filter; $b$ - the Lee filter; $c$ - the Gauss filter; $\mathrm{d}$ - the non-local mean filter and e - the bilateral filter.

The first part of the experiments was aimed at determining the effectiveness of the copy-move detection by the proposed algorithm.

\subsection{Effectiveness of copy-move detection}

The results of experiments aimed at detecting copy-move fragments are shown in Table 1. An example of an image containing copy-move, as well as the result of detecting it using a bilateral filter, is shown in Figures 2 (a) and 2 (b), respectively.

Table 1. F1 metric value of copy-move detection.

\begin{tabular}{|l|c|c|c|c|c|}
\hline \# & Median filter & Lee filter & Gauss filter & $\begin{array}{c}\text { Non-local } \\
\text { mean filter }\end{array}$ & Bilateral filter \\
\hline $\mathbf{1}$ & 0.24 & 0.03 & 0.56 & 0.46 & 0.87 \\
\hline $\mathbf{2}$ & 0.36 & - & 0.43 & 0.64 & 0.93 \\
\hline $\mathbf{3}$ & 0.29 & - & 0.37 & 0.25 & 0.77 \\
\hline $\mathbf{4}$ & 0.44 & 0.02 & 0.42 & 0.33 & 0.69 \\
\hline $\mathbf{5}$ & 0.38 & 0.01 & 0.30 & 0.15 & 0.86 \\
\hline $\mathbf{6}$ & 0.42 & - & 0.14 & 0.14 & 0.87 \\
\hline $\mathbf{7}$ & - & 0.03 & 0.19 & 0.31 & 0.74 \\
\hline $\mathbf{8}$ & 0.49 & - & 0.27 & 0.21 & 0.94 \\
\hline $\mathbf{9}$ & - & - & 0.07 & 0.07 & 0.92 \\
\hline $\mathbf{1 0}$ & 0.53 & 0.01 & 0.86 & 0.00 & 0.92 \\
\hline
\end{tabular}

a)

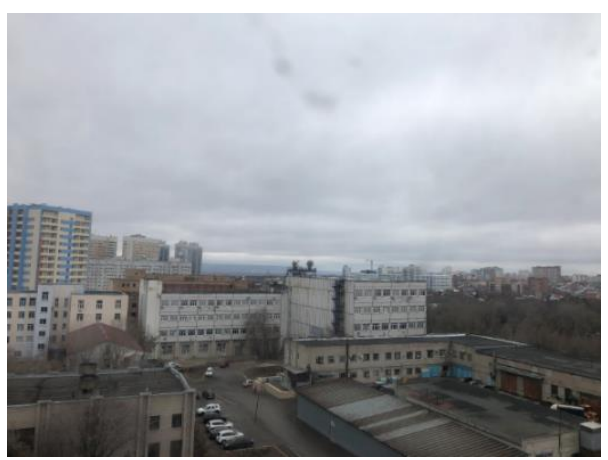

b)

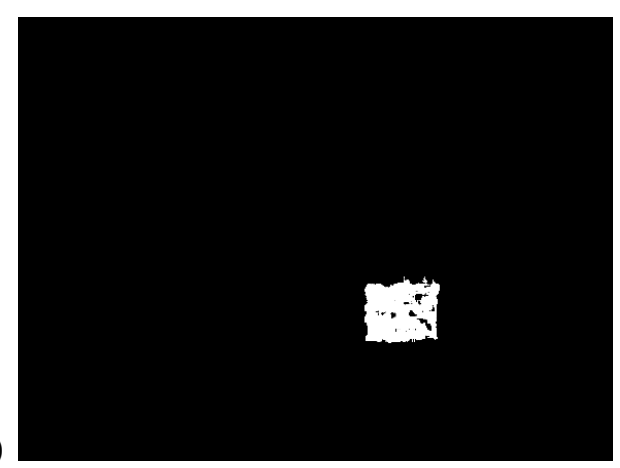

Figure 2. The result of copy-move detection in the image. 


\subsection{Effectiveness of copy-paste detection}

The results of experiments aimed at detecting copy-paste fragments are shown in Table 2. An example of an image containing copy-paste, as well as the result of detecting it using a bilateral filter, is shown in Figure 3 (a) and 3 (b), respectively.

Table 2. F1 metric value of copy-paste detection.

\begin{tabular}{|l|c|c|c|c|c|}
\hline$\#$ & Median filter & Lee filter & Gauss filter & $\begin{array}{c}\text { Non-local } \\
\text { mean filter }\end{array}$ & Bilateral filter \\
\hline $\mathbf{1}$ & 0.25 & - & 0.30 & 0.21 & 0.95 \\
\hline $\mathbf{2}$ & 0.44 & - & 0.43 & 0.15 & 0.96 \\
\hline $\mathbf{3}$ & - & - & 0.54 & 0.28 & 0.87 \\
\hline $\mathbf{4}$ & 0.37 & - & 0.36 & 0.13 & 0.54 \\
\hline $\mathbf{5}$ & 0.22 & - & 0.27 & 0.17 & 0.92 \\
\hline $\mathbf{6}$ & - & - & 0.01 & 0.01 & 0.95 \\
\hline $\mathbf{7}$ & 0.21 & - & 0.26 & 0.30 & 0.91 \\
\hline $\mathbf{8}$ & 0.13 & - & 0.47 & - & 0.86 \\
\hline $\mathbf{9}$ & 0.81 & - & 0.83 & 0.21 & 0.93 \\
\hline $\mathbf{1 0}$ & - & - & 0.39 & 0.17 & 0.83 \\
\hline
\end{tabular}

a)

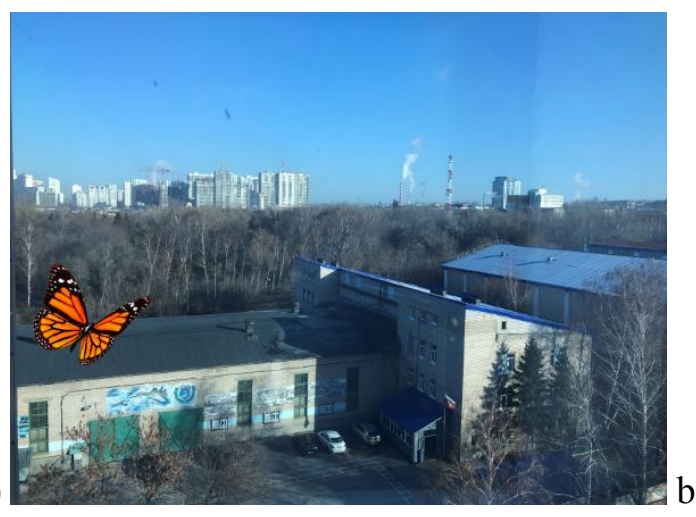

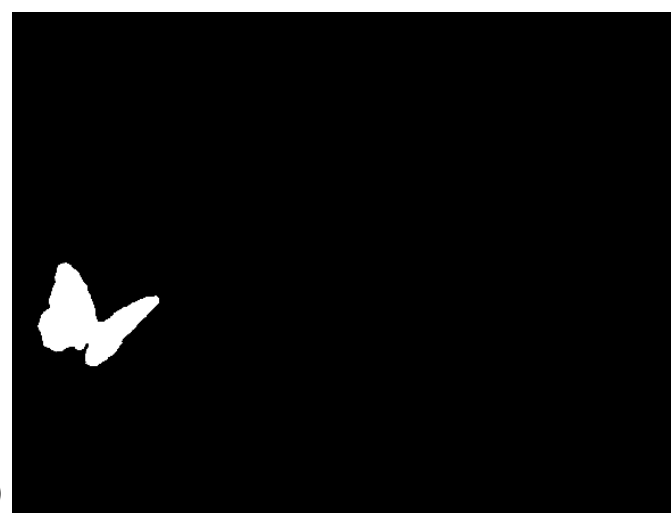

paste detection in the image.

\section{Conclusion}

The image series forgery detection algorithm based on the camera pattern noise analysis has been proposed in the paper. The conducted research has allowed determining the most suitable noise filter in the sense of the selected metric F1 - bilateral filter. Also, experiments have shown the Lee filter is not suitable for solving the problem of copy-move and copy-paste fragments detection. The proposed algorithm allows detecting copy-move fragments with the average F1 value of 0.85 if the bilateral noise filter was used for pattern noise extraction. The average F1 value of 0.87 is reached for copypaste fragments detection with the bilateral noise filter also.

\section{References}

[1] Christian A and Sheth R 2016 Digital Video Forgery Detection and Authentication Technique A Review International Journal of Scientific Research in Science and Technology 2 138-143

[2] Evdokimova N I and Kuznetsov A V 2017 Local patterns in the copy-move detection problem solution Computer Optics 41(1) 79-87 DOI: 10.18287/2412-6179-2017-41-1-79-87

[3] Kuznetsov A V and Myasnikov V V 2016 A copy-move detection algorithm based on binary gradient contours Computer Optics 40 284-293 DOI: 10.18287/2412-6179-2016-40-2-284-293

[4] Kuznetsov A V and Myasnikov V V 2014 A fast plain copy-move detection algorithm based on structural pattern and 2D rabin-karp rolling hash Lecture Notes in Computer Science (including subseries Lecture Notes in Artificial Intelligence and Lecture Notes in Bioinformatics) $\mathbf{8 8 1 4}$ 461-468 
[5] Evdokimova N I and Myasnikov V V 2018 Detecting forgery in image time series based on anomaly detection CEUR Workshop Proceedings 2210 184-192

[6] Hussain M, Chen D, Cheng A, Wei H and Stanley D 2013 Change detection from remotely sensed images: From pixel-based to object-based approaches ISPRS Journal of Photogrammetry and Remote Sensing 80 91-106

[7] Lukáš J, Fridrich J and Goljan M 2006 Detecting digital image forgeries using sensor pattern noise Proceedings of SPIE - The International Society for Optical Engineering $\mathbf{6 0 7 2}$

[8] Fahmy M F and Fahmy O M 2016 A new morphological based forgery detection scheme National Radio Science Conference, NRSC, Proceedings 212-216

[9] Chen M, Fridrich J, Goljan M and Lukáš J 2008 Determining image origin and integrity using sensor noise IEEE Transactions on Information Forensics and Security 3 74-90

[10] Soifer V A, Chernov A V, Chernov V M, Chicheva M A, Fursov V A, Gashnikov M V, Glumov N I, Ilyasova N Y, Khramov A G and Korepanov A O 2009 Computer Image Processing (VDM Verlag Dr. Müller)

[11] Buades A, Coll B and Morel J-M 2005 A non-local algorithm for image denoising Proceedings IEEE Computer Society Conference on Computer Vision and Pattern Recognition, CVPR II 6065

[12] Solomon C and Breckon T 2011 Morphological Processing Fundamentals of Digital Image Processing (John Wiley \& Sons, Ltd) 197-234

\section{Acknowledgments}

This work was supported by RFBR according to the research project № 18-01-00748-a in part of "Introduction" and (2) "Model of a camera's sensor noise" and RF Ministry of Science and Higher Education within the State assignment to the FSRC «Crystallography and Photonics» RAS (Agreement 007-Г3/43363/26) in part of (3) "Forgery detection algorithm" - (4) "Experiments". 